Nonlinear Processes in Geophysics (2005) 12: 291-298

SRef-ID: $1607-7946 / \mathrm{npg} / 2005-12-291$

European Geosciences Union

(c) 2005 Author(s). This work is licensed

under a Creative Commons License.

\title{
Kinetic slow mode-type solitons
}

\author{
K. Baumgärtel ${ }^{1}$, K. Sauer ${ }^{2}$, and E. Dubinin ${ }^{2}$ \\ ${ }^{1}$ Astrophysikalisches Institut Potsdam, Germany \\ ${ }^{2}$ Max-Planck-Institut für Sonnensystemforschung, Katlenburg-Lindau, Germany
}

Received: 2 December 2004 - Revised: 14 January 2005 - Accepted: 16 January 2005 - Published: 11 February 2005

\begin{abstract}
.
One-dimensional hybrid code simulations are presented, carried out in order both to study solitary waves of the slow mode branch in an isotropic, collisionless, medium- $\beta$ plasma $\left(\beta_{i}=0.25\right)$ and to test the fluid based soliton interpretation of Cluster observed strong magnetic depressions (Stasiewicz et al., 2003; Stasiewicz, 2004) against kinetic theory. In the simulations, a variety of strongly oblique, large amplitude, solitons are seen, including solitons with Alfvenic polarization, similar to those predicted by the Hall-MHD theory, and robust, almost non-propagating, solitary structures of slow magnetosonic type with strong magnetic field depressions and perpendicular ion heating, which have no counterpart in fluid theory. The results support the soliton-based interpretation of the Cluster observations, but reveal substantial deficiencies of Hall-MHD theory in describing slow mode-type solitons in a plasma of moderate beta.
\end{abstract}

\section{Introduction}

This work is related to observations presented in 'Slow magnetoacoustic solitons detected by the Cluster spacecraft' by Stasiewicz et al. (2003), hereafter referred to as S. These authors report on the observation of significant magnetic field depressions accompanied with an increase in both plasma density and temperature by the Cluster satellites at the dusk flank of the magnetosphere (Figs. 1, 2 in S). The solitary structures have been seen by the electric and magnetic field instruments as short duration pulses $(\approx 10 \mathrm{~s})$ sweeping with a speed of $250 \mathrm{~km} / \mathrm{s}$ over the slowly moving spacecrafts. The velocity of the structure relative to the background medium could not directly been measured but was estimated to be a fraction of the Alfven velocity. The structures seen in the magnetic field represent deep depressions (up to 85\%) of the ambient field with maximum variation of the field mag-

Correspondence to: K. Baumgärtel

(kbaumgaertel@aip.de) nitude in a direction close to perpendicular to the field direction. The observed features were interpreted in terms of obliquely propagating Hall-MHD solitons of slow magnetoacoustic type. This suggestion was based on a comparison of observed spatial profiles of magnetic field magnitude and density with corresponding numerically calculated soliton profiles. The comparison, which was continued in a subsequent paper (Stasiewicz, 2004), shows good agreement with respect to the depth of the magnetic field depletion and the density increase, but MHD theory predicts a somewhat too small (factor of 2) thickness of the whole structure. Other quantities, such as plasma bulk velocity and ion temperature, were not included in the comparison since the resolution of the ion instruments was not sufficient to get time profiles within the structure.

The aim of this study is to extend the theoretical basis for the interpretation of the observed structures by including kinetic theory. Efforts in this direction appear to be desirable because the measurements were made in a collisionless plasma of moderate ion beta $\left(\beta_{i} \approx 0.25\right)$ whereas the interpretation is given in terms of a fluid plasma model. There is a general reservation to apply fluid theory to finite beta plasmas because of the discrepancies between fluid and kinetic predictions with respect to the properties of smallamplitude waves (Krauss-Varban et al., 1994; Karimabadi et al., 1995). Analytical efforts to incorporate kinetic effects in soliton theory have been focused, so far, on the DNLS equation ('Derivative Nonlinear Schrödinger equation') as one of the most widely used evolution equations for the case of parallel or quasi-parallel propagating MHD wave packets (e.g., Mjolhus and Wyller, 1988; Medvedev and Diamond, 1996). The DNLS equation, however, can be ruled out as basis for our purposes since for conditions considered here (large amplitudes, moderate ion beta and large propagation angle) it is not an appropriate approximation to the superior system of Hall-MHD equations (Baumgärtel, 1999; Ruderman, 2002). Since an analytical treatment of nonlinear phenomena such as solitons on the basis of the VlasovMaxwell system is prohibitively complicated, a numerical 


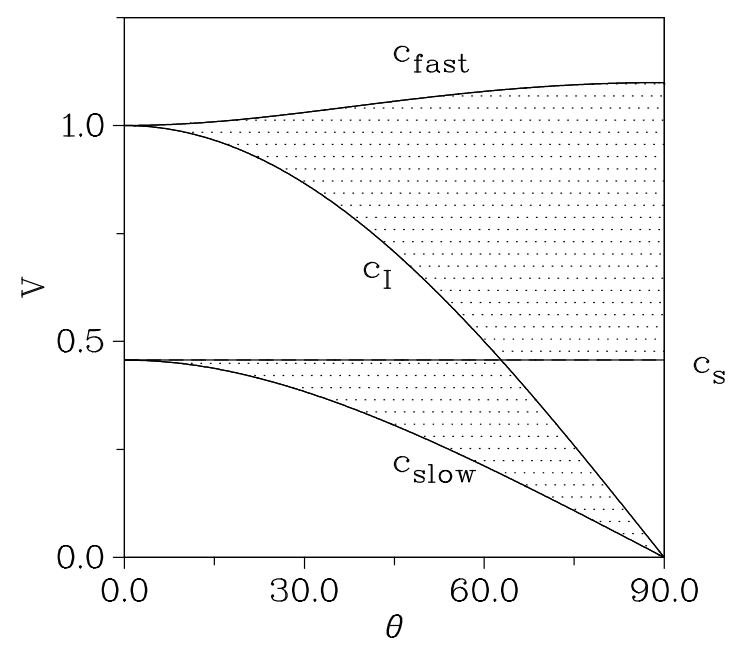

Fig. 1. Constraints on the propagation velocity of Hall-MHD solitons in a $\beta=0.25$ plasma. $V$ is the soliton speed and $\theta$ is the propagation angle relative to the ambient magnetic field. Solitons are permitted in the shaded areas. Upper area: fast-mode solitons; lower area: slow mode solitons. $c_{\text {fast }}, c_{I}, c_{\text {slow }}$ are the propagation speeds in the large wavelength limit $k=0$ of the fast magnetosonic wave, the intermediate (Alfven type) wave and the slow magnetosonic wave, respectively. $c_{s}$ is the ion sound speed. All velocities in units of the Alfven velocity $v_{A} . \beta=p_{k i n} / p_{\text {magn }}=2 / \gamma c_{S}^{2} / v_{A}^{2}$; polytropic equation of state with $\gamma=5 / 3$.

approach is required to incorporate kinetic theory. We employ one-dimensional hybrid code simulations (particle ions, fluid electrons) and show, that in a collisionless, moderate ion- $\beta$ plasma a noticeable variety of obliquely propagating solitons of the slow mode branch may exist, partly not predicted by the Hall-MHD theory. Roughly speaking, we see two primary types of solitons which differ in respect to polarization and propagation velocity. One of them corresponds to the Alfvenic soliton of the fluid theory which turns out to be less sensitive to kinetic effects. The other can be categorized as non-propagating, pressure-balanced structure (PBS), with large-amplitude magnetic depressions, without counterpart in MHD theory. A manifold of solitary slow mode structures may develop in a collisionless plasma which comprise properties of both of the two types. These results may improve our understanding to what extent a fluid treatment is applicable to explain nonlinear phenomena in collisionless plasmas.

\section{Recall: fluid results on slow mode solitons}

In this preliminary section we remind briefly on some results of the fluid theory with respect to properties of obliquely propagating Hall-MHD solitons of the slow mode branch. In order to examine solitary solutions of the Hall-MHD equations one may proceed in two distinct ways. A rather direct way uses the stationary wave approximation of the basic system (all variables depend only on $x-V t$ ), from which solitary waves can be isolated with the help of the fixed-point- analysis (Hau and Sonnerup, 1991; Baumgärtel et al., 1997; McKenzie and Doyle, 2002). An alternative method derives first an evolution equation from the superior system, containing nonlinearity and dispersion only to lowest order, and tries to apply inverse scattering theory in order to find 'genuine' solitons. The most general evolution equation that has been derived from the Hall-MHD system, the DNLS equation, describes preferentially quasi-parallel, small-amplitude perturbations in a low- $\beta$ plasma. It was recently shown by Ruderman (2002) that the DNLS covers oblique wave evolution with arbitrary amplitudes as well, confirming an early suggestion of Kennel et al. (1989). It does not, however, apply to the parameter combination reported in $\mathrm{S}$ (large amplitudes, strong obliquity, moderate $\beta$ ). The fixed-point analysis reveals, that the soliton speed $V$ relative to the plasma is constraint to areas not accessible for any of the small-amplitude wave types for any real wave number ('gaps'). Figure 1 depicts regions of permission in the $V-\theta$-plane (shaded areas) of solitons in a $\beta=0.25$ plasma. It is seen from this figure that for large propagation angles the slow mode soliton velocity may vary between the speeds $c_{\text {slow }}$ of the slow magnetosonic wave and $c_{I}=v_{A} \cos \theta$ ( $v_{A}$ Alfven velocity) of the intermediate wave, both in the $k=0$ limit. It should be pointed out here that McKenzie and Doyle (2002) erroneously calculated the extended interval $c_{\text {slow }}<V<c_{s}$ as constraint for the velocity of slow mode solitons. With respect to polarization, the soliton exhibits elements of both the slow magnetosonic and the intermediate wave. Thus, the slow mode soliton generally is associated with pressure perturbations as well as noncoplanar variations of magnetic field and plasma velocity. The character of the soliton, however, especially the magnetic field variation across the structure, changes markedly within the region of permission $c_{\text {slow }}<V<c_{I}$. This is illustrated in Fig. 2 which depicts shapes for different types of solitons.

Figure 2a shows a soliton with a polarization like that of a slow magnetosonic wave (magnetic vector changes in magnitude rather than in direction, 'cigar-like' shape of the $B_{y}-B_{z}$ phase plot). When the soliton speed approaches the lower limit $c_{\text {slow }}$, the amplitude rapidly decays and simultaneously the polarization tends to become linear. In the opposite limit $V \rightarrow c_{I}$ the soliton approaches a quite different state, which is depicted in Fig. 2c. Unlike the former case, neither the amplitude decreases nor the polarization becomes linear, as dispersion theory requires for an oblique, smallamplitude Alfven wave. Instead, the magnetic vector rotates once around the propagation direction with little variation in magnitude ('pancake-like' phase plot), associated with largeamplitude variations in the transverse bulk velocity components $u_{y}$ and $u_{z}$. The soliton size increases and approaches a value of about $10 c / \omega_{p i}$. The whole structure resembles a finite-amplitude, obliquely propagating, nearly circularly polarized, Alfven wave packet. It might be alternatively categorized as a special case of a rotational discontinuity, in which the magnetic vector undergoes a full rather than half a rotation. Since the magnetic field depression remains moderate, these Alfvenic solitons are not in the focus of interest 
(a)

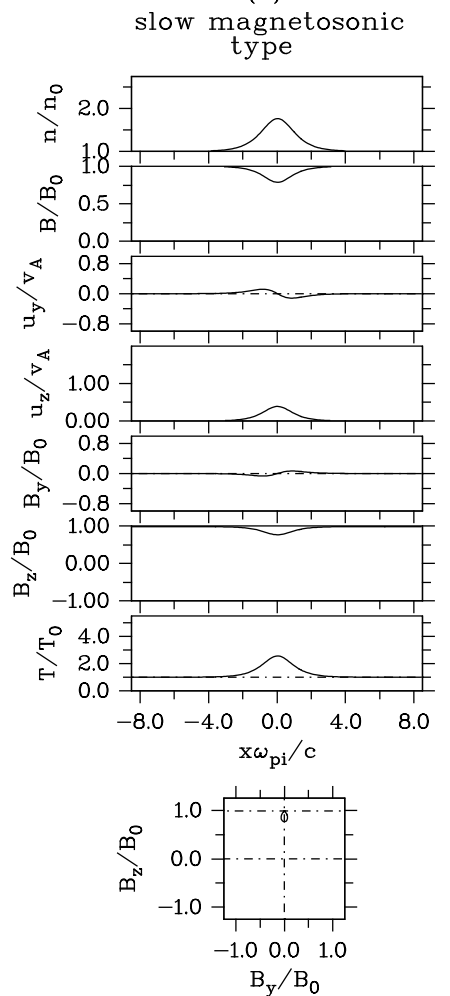

(b) maximum field depresssion
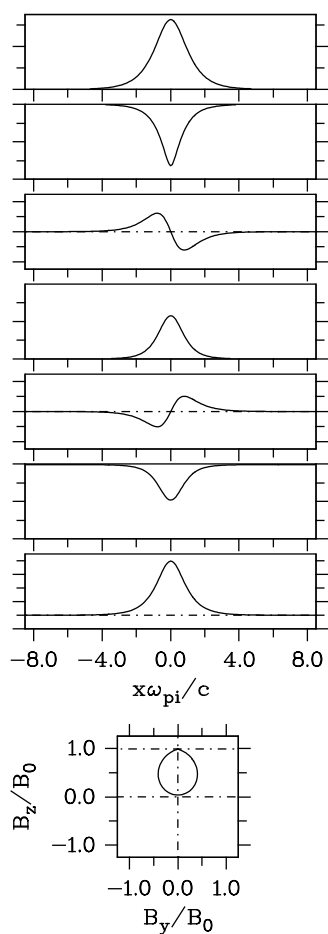

(c) Alfvenic type
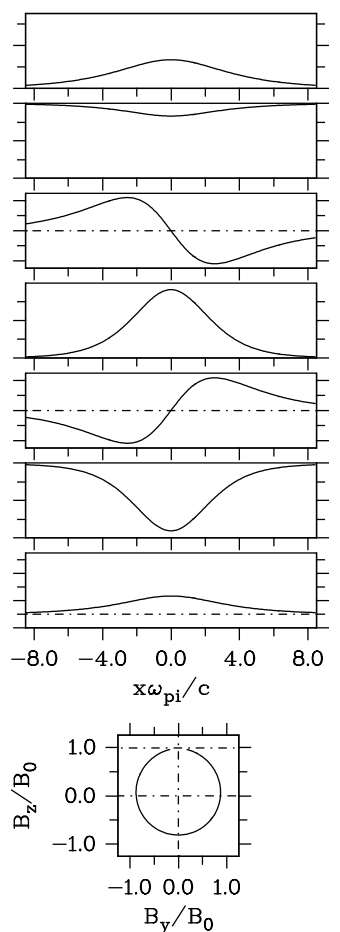

Fig. 2. Examples of magnetically rarefactive Hall-MHD solitons of the slow mode branch in a $\beta=0.25$ plasma, propagating at an angle of $\theta=80^{\circ}$ relativ to the ambient magnetic field in positive x-direction. According to Fig. 1 the soliton speed V is constraint to the interval $c_{\text {slow }}<V<c_{I}$ (here $c_{\text {slow }} \approx 0.072 v_{A}, c_{I} \approx 0.174 v_{A}$ ). (a) Soliton of slow magnetosonic type, $V \approx 0.1 v_{A}$, size $\approx 4 c / \omega_{p i}$. (b) Soliton having approximetely the largest possible magnetic depression, $V \approx 0.143 v_{A}$, size $\approx 4 c / \omega_{p i}$. (c) Soliton of Alfvenic type, $V \approx 0.17 v_{A}$, size $\approx 10 c / \omega_{p i}$. The profiles result from numerical integration of the fully nonlinear Hall-MHD equations in stationary wave approximation, using the fixed-point technique. Left-propagating solitons differ from those above in the sign of $u_{x}$ (not shown), $u_{z}$, and $B_{y}$.

in the context of the discussions in $\mathrm{S}$. The soliton shown in Fig. $2 b$ has been selected from the one-parameter manifold by looking for a most large magnetic field depression. It is almost identical with that shown in Fig. 3 in S. Since the transverse component $B_{y}$ generally goes through zero in the center of the soliton, the maximum depression is realized in the case when $B_{z}$ reaches zero, leading to $B=B_{x}=B_{0} \cos \theta$. Figure $2 \mathrm{~b}$ shows a case close to this situation. Note that the size in this case is less than half of that of the Alfvenic soliton in Fig. 2c.

\section{Kinetic slow mode-type solitons}

\subsection{Hall-MHD versus kinetic theory: two examples}

The first point we wish to make is to show that only a small fraction of the manifold of oblique slow mode solitons, predicted by the fluid model, survive in kinetic theory. To demonstrate this, we set up MHD-predicted spatial soliton profiles as initial conditions in a hybrid code simulation and permit the system to evolve in time selfconsistently. A few details concerning the simulations are given in the Appendix. Figure 3 illustrates what happens to the Alfvenic fluid soli- ton of Fig. 2c when launched in a collisionless plasma (same propagation angle and ion- $\beta$ ). The soliton has been specified as left-propagating; in order to keep it longer in the simulation box, the plasma is streaming as a whole with a velocity of $0.165 v_{A}$ in positive $\mathrm{x}$-direction. It is seen from the time sequence plots of $B$ and $n$ in Fig. 3 that this soliton is little affected and not in conflict with kinetic theory. It appears as stable, long-lived structure, maintaining its shape until the end of the run $\left(t=2000 \Omega_{i}^{-1}\right)$. Kinetic modifications are not fundamental in nature and concern the ion temperature evolution: while a somewhat reduced peak in the perpendicular temperature $T_{i \perp}$ is maintained, the parallel temperature $T_{i \|}$ rapidly relaxes to the equilibrium value, leaving an ion temperature anisotropy $T_{i \perp} / T_{i \|}>1$ inside the soliton.

Unlike the former case, the soliton with maximum field depression (Fig. 2b) does not survive in the simulation. This is demonstrated in Fig. 4. As seen from the stackplots of $B$ and $n$ in Fig. 4, the soliton rapidly degenerates to a smallamplitude perturbation which is not of further interest. The only features worth to mention are a splitting into two events, each of them associated with an increase in the perpendicular ion temperature (not shown), and the emission of two rightpropagating intermediate wave pulses (not shown). At a first 

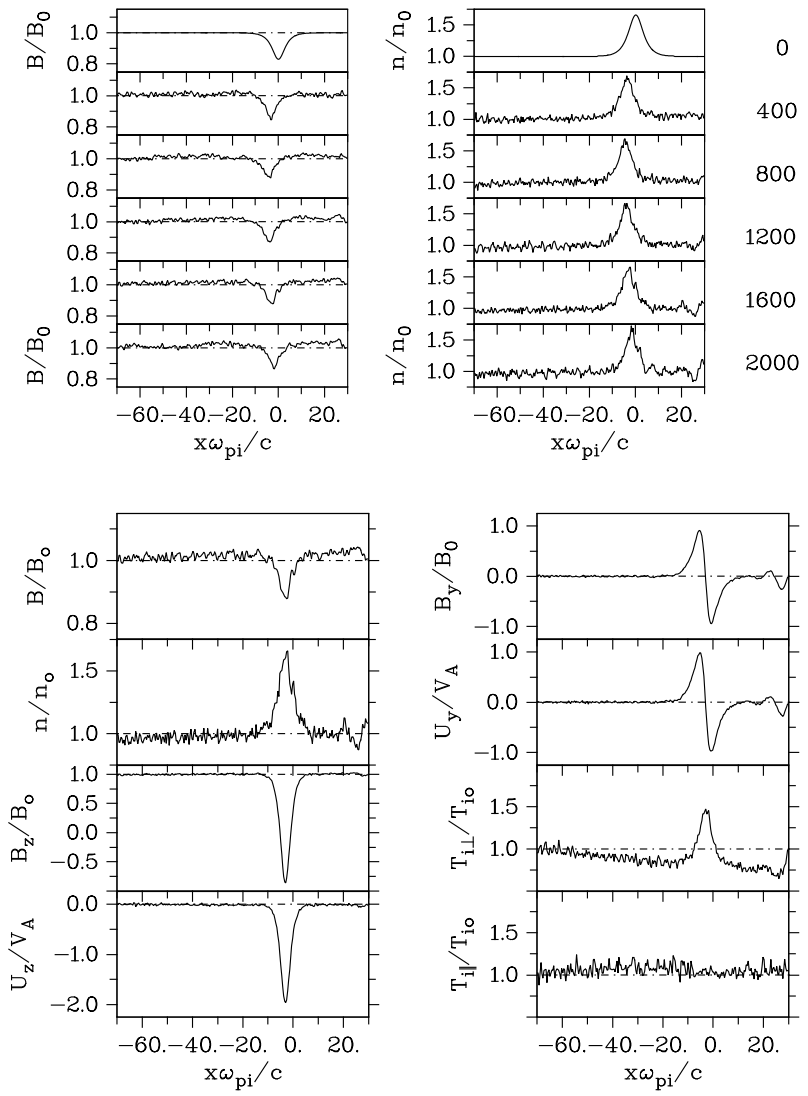

Fig. 3. Space-time evolution of the Alfvenic fluid soliton of Fig. 2c when launched in negative $x$-direction in a collisionless plasma with isotropic Maxwellian ion distribution $\left(\beta_{i}=0.25, \beta_{e}=0.025 ; \theta=80^{\circ}\right)$ and a bulk speed of $0.165 v_{A}$ in positive $\mathrm{x}$-direction, as a result of a hybrid code simulation. Shown are stackplots of $B, n$ (top) and profiles of relevant quantities at the time $t=2000 \Omega_{i}^{-1}$ (bottom). The soliton is almost standing in the simulation box, i.e., in the plasma frame it travels with $V \approx 0.165 v_{A}$ to the left.

look, this behaviour might rise doubts on the qualification of fluid predicted solitons to describe solitary phenomena in a collisionless plasma.

\subsection{Initial conditions/mechanisms used to generate solitons}

Our second point is to stress that a collisionless plasma, in addition to the Alfvenic soliton, does admit a variety of largeamplitude, solitary structures with a polarization of the slow magnetosonic type, not predicted by the fluid model. In the following we illustrate general aspects of the evolution and the properties of such kinetic solitons with a few specific cases in a series of hybrid simulations. As we have seen above, fluid solitons are not necessarily appropriate as initial conditions in the simulations for this purpose. Since we do not know the criteria for an arbitrary initial condition to generate solitons during the time evolution, we try several artificial initial states which have in common a local-
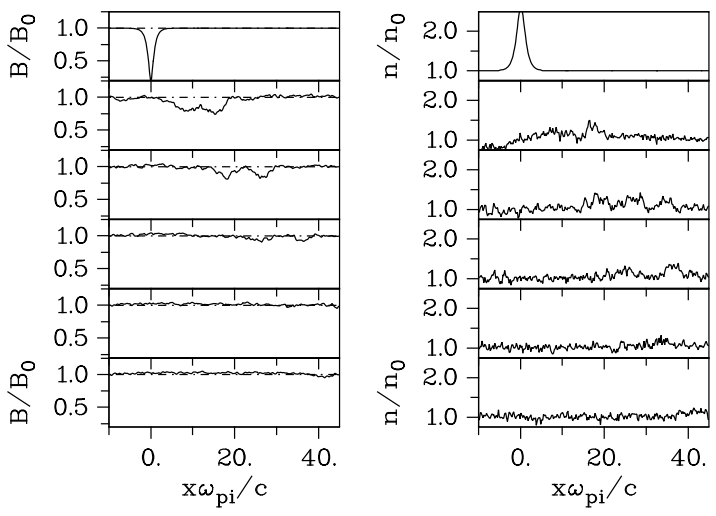

$\mathrm{t} \Omega_{\mathrm{i}}=$

0

100

200

300

400

500

Fig. 4. Space-time evolution of the fluid soliton of Fig. 2b, with maximum field depression, when launched in a collisionless plasma; same $\beta_{i}, \beta_{e}$, and $\theta$ as in Fig. 3. Shown are stackplots of $B$ and $n$.

ized perturbation of an otherwise uniform plasma. Thereby, we do not address the question whether or not such initial states/generation mechanisms may arise in nature. First, we study the space-time evolution of two initial perturbations with a polarization similar to that of the fluid soliton in Fig. 2b. Second, we study the plasma response to two alternative perturbation mechanisms: we run a simulation in which ions are locally injected into the uniform plasma during a time period, and we consider a case in which the plasma is locally perturbed by an external double-sheet current. In all runs solitary structures develop, illustrating the manifold and the robustness of kinetic slow mode solitons. At the same time, the particular role of the non-propagating (or almost non-propagating) structures, which emerge in all of the runs with nearly the same features, becomes evident.

\subsection{Initial conditions with large field depressions}

The first example, illustrated in Fig. 5, depicts stack plots of $B$ and $n$ (top) giving the evolution to $t=1000 \Omega_{i}^{-1}$ of an initial structure which corresponds to the fluid soliton in Fig. $2 b$, stretched in space by a factor of 8 . Subsequent to a transient deformation of the initial profile, two solitonlike entities emerge self-consistently after about $300 \Omega_{i}^{-1}$. One of them propagates to the right with a velocity near to $V=c_{I}$, maintaining its shape almost until the end of the run. It exhibits typical features of the Alfvenic soliton in Fig. 3 (size $\approx 10 \omega_{\text {pi }} / c$, moderate field depletion, significant $B_{y^{-}}$and transverse plasma bulk velocity variations, density increase by a factor $\approx 2$ ) and can be categorized correspondingly. The increased parallel ion temperature around the soliton is a remnant of the initial peak in $T_{i}$ which disperses with time and bears little relationship to the soliton. The second solitary structure seen in Fig. 5 appears as quite different event. It persists almost at the position of generation and exhibits a much stronger magnetic field depletion. Variations of the non-coplanar component $B_{y}$ are absent, perturbations 

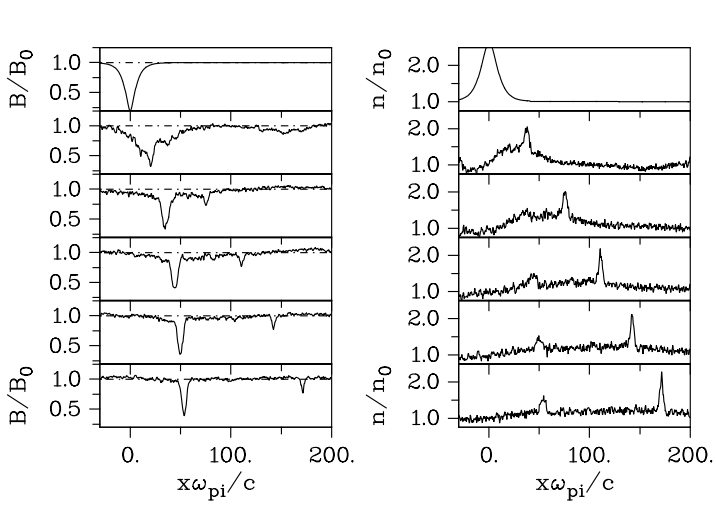

$\mathrm{t} \Omega_{\mathrm{i}}=$

200

400

600

800

1000
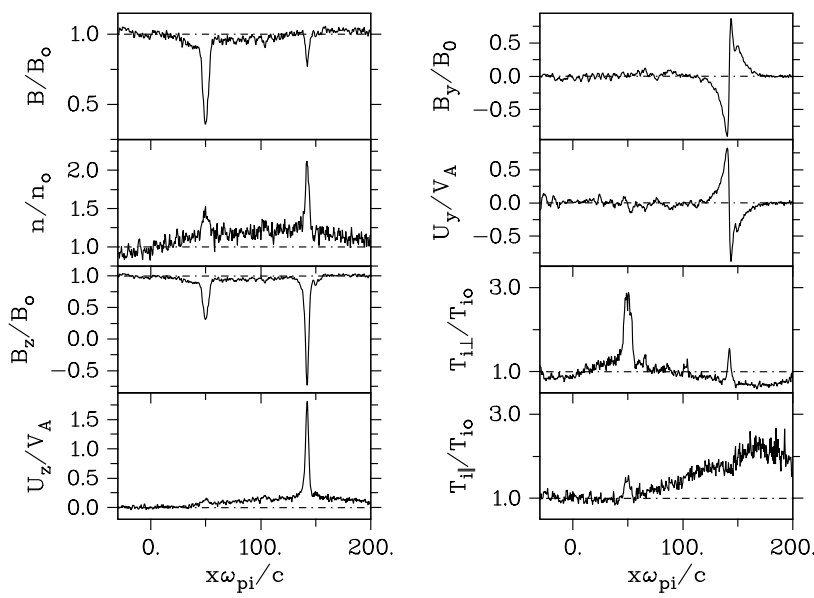

Fig. 5. Soliton formation in a collisionless plasma (same $\beta_{i}, \beta_{e}$, and $\theta$ as in Fig. 3) as a result of a hybrid code simulation. The initial condition represents the fluid soliton of Fig. $2 b$ streched in space by a factor of 8 . Shown are stackplots of $B, n$ (top) and profiles of relevant quantities at the time $t=800 \Omega_{i}^{-1}$ (bottom).

of the plasma bulk velocity are close to the thermal level, and there is only a moderate density signal. A notable feature is the development of a significant ion temperature anisotropy $T_{i \perp} / T_{i \|}>1$ inside the soliton. The perpendicular temperature $T_{i \perp}$ is increased by a factor of $\approx 3$ while the parallel temperature $T_{i \|}$ remains almost unaffected. Thus, the ions show a distinct departure from their initial Maxwellian property in the region of depleted field. This soliton represents an almost pressure-balanced structure (PBS) in which the increased ion temperature $T_{i \perp}$ rather than the density hump balances the magnetic pressure deficit. This solitary structure is not predicted by Hall-MHD theory, thus we are confronted here with a kinetic phenomenon.

The initial configuration whose space-time evolution is depicted in Fig. 6 is set up again by a broadened version of the soliton in Fig. 2b, here realized by the choice of a higher ion$\beta\left(\beta_{i}=1\right.$ instead of $\left.\beta_{i}=0.25\right)$. It is seen from Fig. 6 that a slow mode soliton is launched from this initial perturbation, propagating to the right with a speed less than that of the Alfven-type soliton in Fig. 5. It is neither accompanied by a $B_{y}$-variation nor has it the character of a strictly pressurebalanced structure. The peak in $T_{i \perp}$ gives evidence of per-
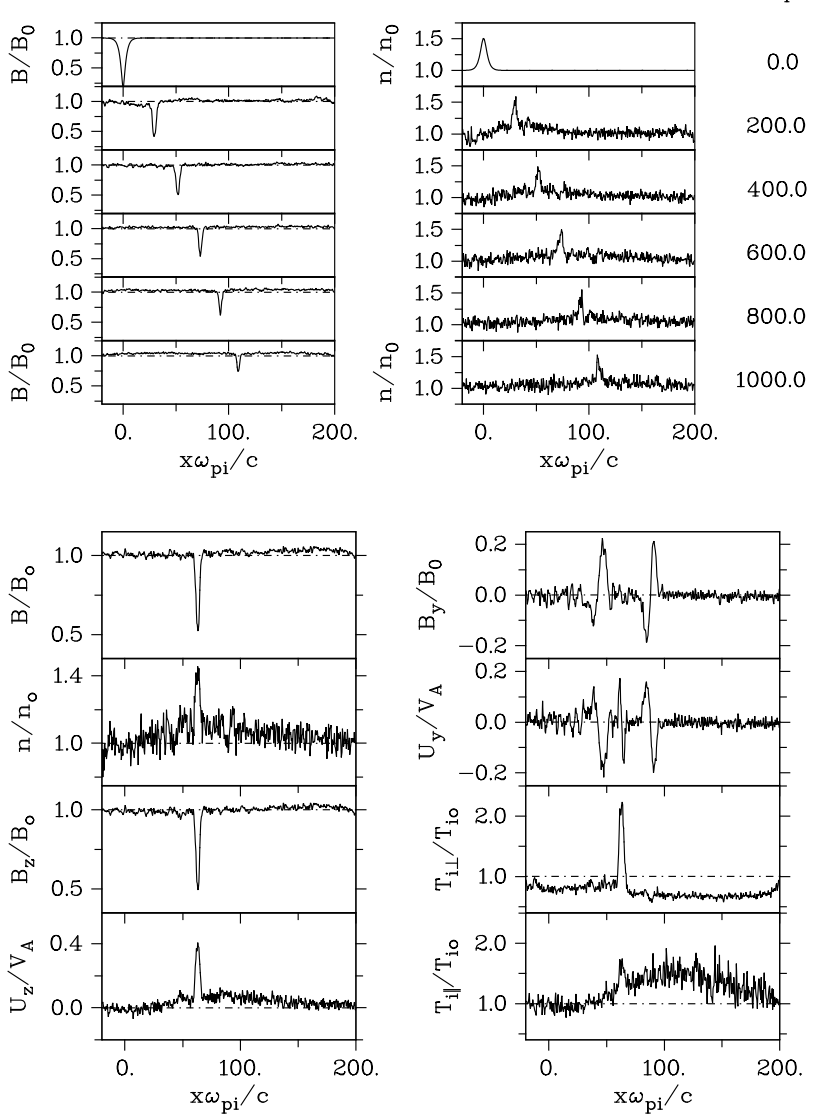

Fig. 6. The same as Fig. 5; the initial condition represents again a 'broadened' version of the soliton in Fig. 2b, realized here by the choice $\beta=1$ instead of $\beta=0.25$. Stack plots of $B$ and $n$ (top) and profiles of relevant qantities at the time $t=500 \Omega_{i}^{-1}$ (bottom).

pendicular ion heating inside the soliton, i.e., it appears as a moving version of the PBS soliton in Fig. 5. Note, however, that the amplitude decays with time, likely caused by the motion relative to the background plasma, which disqualifies this soliton as a long-lived structure. The perturbations in $B_{y}$ and $u_{y}$ adjacent to the soliton location, seen in the bottom panels of Fig. 6, represent two right-propagating intermediate wave pulses launched in addition to the soliton.

\subsection{Soliton generation by mass loading}

The third example, in which ions are locally injected into the simulation box, is illustrated in Fig. 7. Injection takes place around $x=0$ within the period $0<t<200 \Omega_{i}^{-1}$ at a rate of $\approx 10$ particles/time step with a velocity distribution consistent with the initial particle distribution. From the stack plots of $B$ and $n$ it is seen, that, after initial transient processes, a non-propagating, large-amplitude solitary structure evolves with similar properties as that in Fig. 5. Size and depth of the magnetic depression as well as the spatial variation of density and temperatures in the final time are quite similar. We point to the variation of the non-coplanar component $u_{y}$ of the plasma bulk velocity, which is clearly related to the 

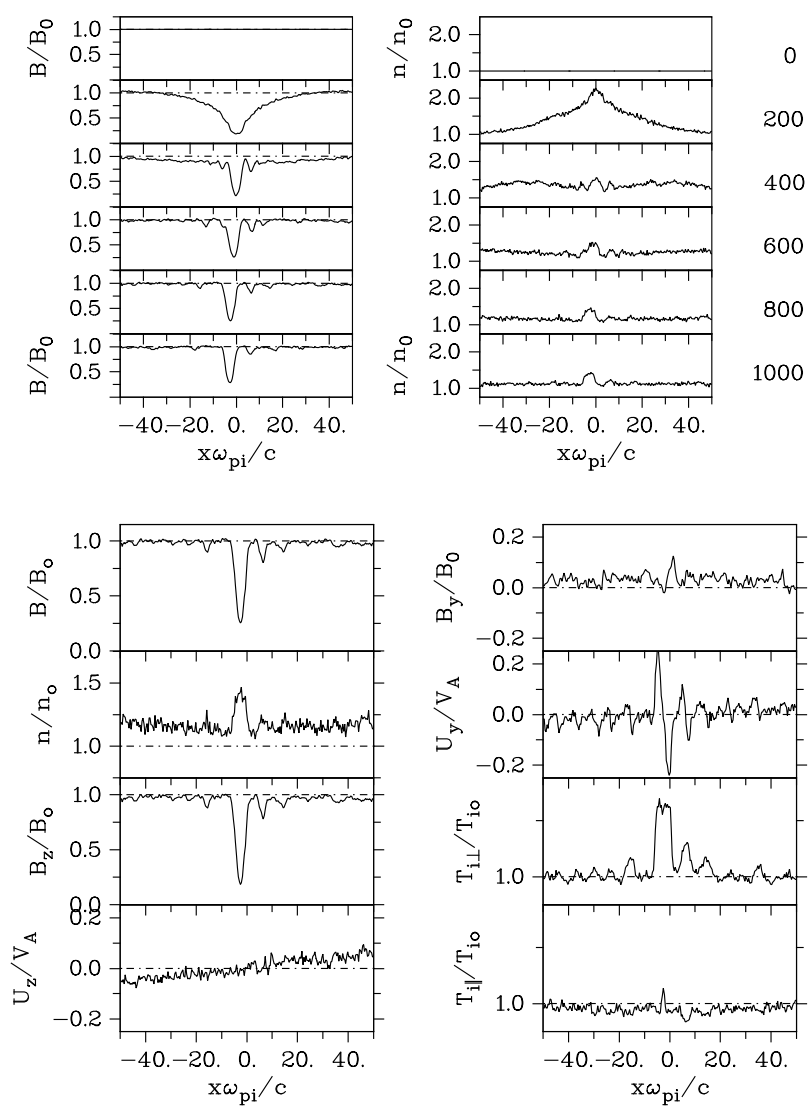

Fig. 7. Generation of a non-propagating magnetically rarefactive solitary structure by particle injection into a uniform Maxwellian plasma (same $\beta_{i}, \beta_{e}$, and $\theta$ as in Fig. 3). Around $x=0$ ions are continuously injected at a rate $\approx 10$ particles/time step for a time priod $0<t<200 \Omega_{i}^{-1}$ with a velocity distribution consistent with the initial ion distribution. Stack plots of $B$ and $n$ (top) and profiles of relevant quantities (bottom) at the end of the run $\left(t=1000 \Omega_{i}^{-1}\right)$.

soliton. It indicates the presence of a current in y-direction, necessary to maintain the $x$-variation of $B_{z}$ in a pressurebalanced structure. Again, perpendicular ion heating occurs within the depleted field region. Since the simulation has started in this case with uniform, isotropic ion temperature across the box, evidence is given that the formation of the soliton is inherently coupled with perpendicular ion heating. The mechanism responsible for this heating has not yet been explained.

\subsection{Soliton generation by an external current}

In the fourth case, the plasma is transiently excited by an external double-sheet current along the y-direction, located at the center of the simulation box. The $\mathrm{x}$-variation of the current is prescribed as derivative of a Gaussian profile. After a time period of $100 \Omega_{i}^{-1}$ the current is switched off and the subsequent evolution proceeds undriven. This example is illustrated in Fig. 8. From the stack plots it is seen that an almost non-propagating soliton evolves after transient pro-
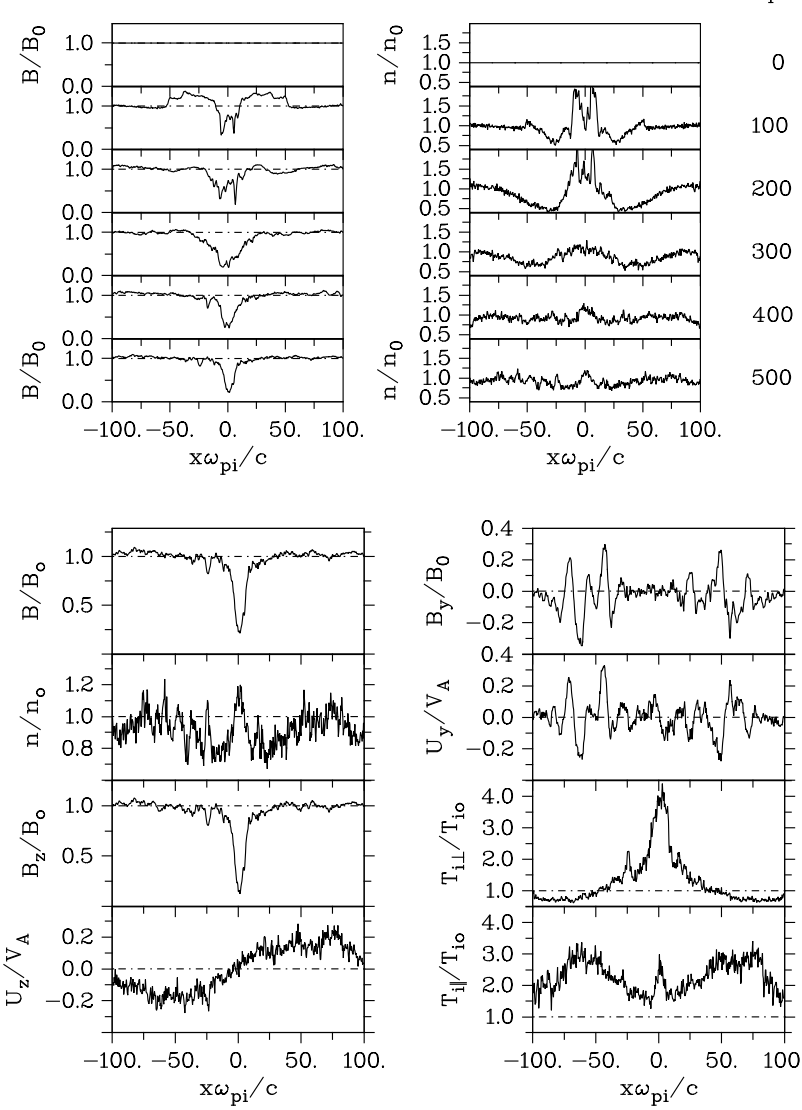

Fig. 8. Soliton formation subsequent to a transient, localized plasma perturbation by an external double-sheet like current. The $\mathrm{x}$-variation of the external current is $J_{y} / e n_{0} v_{A}=a \sqrt{2} x / H \exp \left(-x^{2} / H^{2}\right)$ with $a=-0.2, H=10 c / \omega_{p i}$. The current is at work until $t=50 \Omega_{i}^{-1}$. Stack plots of $B$ and $n$ (top) and profiles of relevant quantities (bottom) at the end of the run $\left(t=500 \Omega_{i}^{-1}\right)$. Same $\beta_{i}, \beta_{e}$, and $\theta$ as in Fig. 3 .

cesses, the properties of which are almost the same as in the previous cases. Linearly polarized intermediate wave pulses are also launched to both sides of the source, as seen from the $B_{y}$ and $u_{y}$ profiles. They appear to be associated with increases in the parallel ion temperature. In an additional run, the plasma was exposed to the same current with opposite directions, which tends to create a magnetic hump instead of a dip. The magnetic compression, however, rapidly relaxed after switching off the current, indicating that it does not represent a stable structure.

\section{Discussion and conclusions}

We have examined the evolution of magnetically rarefactive, large-amplitude, slow mode solitons propagating oblique to the ambient magnetic field $\left(\theta=80^{\circ}\right)$ in a collisionless plasma of moderate ion- $\beta$ ( $\left.\beta_{i}=0.25\right)$ via 1 -D hybrid code simulations. The study is partly motivated by the goal to test the fluid-based theoretical model for the explanation of 
solitary magnetic depressions, observed by the Cluster satellites (Stasiewicz et al., 2003; Stasiewicz, 2004), against kinetic theory. The simulations give evidence that magnetically rarefactive solitary structures of quite different properties may exist in a collisionless plasma, and that they may be generated under different circumstances. The spectrum includes Alfvenic solitons, similar to those predicted by the HallMHD theory as well as non-propagating, pressure-balanced, solitary structures without counterpart in fluid theory. Characteristic feature of the latter are a large-amplitude magnetic depression, accompanied with a moderate density enhancement, a variation of the non-coplanar plasma bulk velocity component $u_{y}$ and an enhanced perpendicular ion temperature, causing a significant ion temperature anisotropy in the depleted field region. The mechanism which energizes ions perpendicular to the magnetic field inside of PBS-type solitons remains to be explained. The variation of the plasma bulk velocity component $u_{y}$ indicates the presence of a current which is necessary to maintain the $x$-variation of $B_{z} \approx B$. A moving version of this soliton type is subject to damping in amplitude. The tendency of a collisionless plasma to form almost non-propagating regions of depleted magnetic field is clearly a kinetic effect. It becomes as more dominant, as larger the ion- $\beta$ is, and may explain the frequent occurrence of 'magnetic holes' in high- $\beta$ plasmas (Baumgärtel et al., 2003). The fact that a soliton possessing the polarization of the slow magnetosonic wave appears as robust structure in the simulations is seemingly in contradiction to the expections one might have from linear kinetic wave theory, which predicts strong collisionless damping for the slow magnetoacoustic wave. One should be aware, however, of the essential non-propagating nature of this soliton, which suppresses wave-particle interaction and thus enables the establishment of a stable structure. This suggestion is supported by the observation of damping in cases of finite propagation velocity of this type of solitons in the simulations.

Solitary slow mode structures in a $\beta_{i}=0.25$ plasma appear in the simulations generally with a size of $8-10 c / \omega_{p i}$, exceeding the fluid predicted size for maximum amplitude $\left(\approx 4 c / \omega_{p i}\right.$ in Fig. 2 b) by a factor of 2 .

As far as the comparison of the simulation results with the observations is concerned, the situation is as follows. The larger size of kinetic solitons of all types fits well into the observations. Alfvenic solitons likely can be ruled out as candidates because of their less pronounced field depression. On the other hand, standig or almost non-propagating PBS-type solitons are not associated with a sufficiently strong density signal. Events which combine strong field depressions and strong density enhancement have been seen in the simulations only as transient structures. Since measurements of ion quantities such as plasma bulk velocity and ion temperature inside the magnetic depressions are not available, the most striking kinetic prediction, the ion temperature anisotropy within the soliton, cannot be confirmed by the observations reported in S. The same is true with respect to the bulk velocity variations which could give information on the physical nature of the events. There are, however, indications of per- pendicular ion heating in previous reports on large-amplitude magnetic depressions of quite different spatial scales (Winterhalter et al., 1994; Fränz et al., 2000; Tsurutani et al., 2002)). There is one remaining point which might contribute to clarify the character of the observed solitary structures on the basis of the magnetic field data, an inspection of the $B_{y}$ variation across the events. It could give indications whether PBS-like or Alfvenic solitary structures have been observed.

In order to test the influence of a moderate anisotropy of the equilibrium plasma on the soliton formation, some of the runs have been repeated with a Bi-Maxwellian ion velcity distribution below the threshold of the Mirror instability $\left(T_{i 0 \perp} / T_{i 0 \|}=1.4\right)$ loaded initially into the simulation box. No significant changes have been seen in the space-time evolution; the solitary structures appeared almost unaffected by the anisotropy.

One may list the basic findings with respect to strongly oblique propagating, kinetic, solitons of the slow mode branch as follows: (1) Only the Alfvenic soliton of the Hall-MHD theory survives in kinetic theory; (2) a collisionless plasma admits non-propagating, pressure-balanced, solitons with large magnetic depressions, moderate density signals and a strongly enhanced perpendicular ion temperature within the structure; (3) the size of kinetic solitons in a plasma with $\beta_{i}=0.25$ is generally around 10 ion skin lengths; (6) a moderate ion temperature anisotropy $T_{i 0 \perp} / T_{i 0 \|}>1$ below the threshold of the Mirror instability has little influence on the formation and the properties of solitons.

In summary, kinetic effects have been shown to contribute significantly to the appearance of oblique, slow mode-type solitons in a $\beta_{i}=0.25$ plasma. Our results lead us to argue that caution is required when Hall-MHD theory is applied to explain nonlinear phenomena of slow mode character in collisionless plasmas even for moderate beta.

\section{Appendix}

The one-dimensional hybrid simulations employ a system with the simulation axis along $\mathbf{x}$ and the undisturbed magnetic field $\mathbf{B}$ in the $\mathrm{x}$-z-plane (angle $\theta$ relative to the $\mathrm{x}$ axis). Simulations are typically performed using simulation boxes with a number of cells such that the ion skin length $c / \omega_{p i}$ is resolved more than twice (typical cell size $\left.\sim 0.4 c / \omega_{p i}\right), 200-400$ protons per cell, and a time step of $0.05 \Omega_{i}^{-1}$ ( $\omega_{p i}$ proton plasma frequency, $\Omega_{i}$ proton gyrofrequency) and have been typically carried out until $1000 \Omega_{i}^{-1}$. The background plasma is isotropic with a Maxwellian proton distribution $\left(\beta_{i}=0.25\right)$ and an adiabatic, massless, colder electron fluid $\left(\beta_{e}=0.025\right)$. A few runs have been started with an anisotropic (Bi-Maxwellian) proton distribution function. 'Free' boundary conditions are used in the sense that particles are continuously injected at both boundaries of the simulation box from a buffer zone filled with ions having a velocity distribution consistent with the initial particle distribution, to balance particles escaping from the box. 
Acknowledgements. The authors wish to express their gratitude to the International Space Science Institute (ISSI) at Bern, where this work was initiated, for their support of the collaboration in the framework of the Visiting Science Program. The authors would like to thank K. Stasiewicz for making accessible Cluster magnetic field data presented and discussed in the PRL paper 2003.

Edited by: J. Büchner

Reviewed by: E. Mjølhus and another referee

After submitting the manuscript an additional inspection of the Cluster magnetic field data discussed in $\mathrm{S}$ revealed that variations of the non-coplanar magnetic field component are almost absent during the events S1, S2, S3 in Fig. 2 of S (data courtesy K. Stasiewicz). This supports the suggestion that the events under discussion likely represent PBS-type kinetic solitons rather than conventional Hall-MHD solitons.

\section{References}

Baumgärtel, K.: Soliton approach to magnetic holes, J. Geophys. Res., 104, 28 295-28 308, 1999.

Baumgärtel, K., Dubinin, E., Sauer, K., and Story, T. R.: Solar wind magnetic holes: signatures of slow mode-type MHD solitons?, Adv. Space Res., 20, 69-74, 1997.

Baumgärtel, K., Sauer, K., and Dubinin, E.: Towards understanding magnetic holes: Hybrid simulations, Geophys. Res. Lett., 30, doi:10.1029/2003GL017373, 2003.

Fränz, M., Burgess, D., and Holbury, T. S.: Magnetic field depressions in the solar wind, J. Geophys. Res., 105, 12725-12732, 2000.

Hau, L. N. and Sonnerup, B. U. Ö.: Self-consistent gyroviscous fluid model of rotational discontinuities, J. Geophys. Res., 96, $15767-15778,1991$.

Karimabadi, H., Krauss-Varban, D., and Omidi, N.: Characteristic speeds in high $\beta$ isotropic/anisotropic plasmas, Phys. Plasmas, 2, 4177-4184, 1995.
Kennel, C. F., Buti, B., Hada, T., and Pellat, R.: Nonlinear, dispersive, elliptically polarized Alfven waves, Physics Fluids, 31, 1949-1989, 1989.

Krauss-Varban, D., Omidi, N., and Quest, K. B. Mode properties of low-frequency waves: Kinetic theory versus Hall-MHD, J. Geophys. Res., 99, 5987-6009, 1994.

McKenzie, J. F. and Doyle, T. B.: The properties of fast and slow oblique solitons in a magnetized plasma, Phys. Plasmas, 9, 5563, 2002.

Medvedev, M. V. and Diamond, P. H.: Fluid models for kinetic effects on coherent nonlinear Alfven waves, J. Plasma Phys., 3, 863-873, 1996.

Mjolhus, E. and Wyller, J.: Nonlinear Alfven waves in a finite beta plasma, J. Plasma Phys., 40, 299-310, 1988.

Ruderman, M. S.: DNLS equation for large-amplitude solitons propagating in an arbitrary direction in a high- $\beta$ Hall plasma, J. Plasma Physics 67, 271-276, 2002.

Stasiewicz, K.: Theory and observations of slow-mode solitons in space plasmas, Phys. Rev. Lett., 93, doi:10.1103/PhysRevLett.93.125004, 2004.

Stasiewicz, K., Shukla, P. K., Gustavson, G., Buchert, S., Lavraud, B., Thide, B., and Klos, Z.: Slow magnetoacoustic solitons detected by the Cluster spacecraft, Phys. Rev. Lett., 90, 085002-14, 2003.

Tsurutani, B. T., Dasgupta, B., Galvan, C., Neugebauer, M., Lakhina, G. S., Arballo, J. K., Winterhalter, D., Goldstein, B. E., and Buti, B.: Phase-steepened Alfven Waves, proton perpendicular energetization and the creation of magnetic holes and magnetic decreases: The ponderomotive force, Geophys. Res. Lett., 29(24), 2233, doi:10.1029/2002GL015652, 2002.

Winterhalter, D., Neugebauer, M., Goldstein, B. E., Smith, E. J., Bame, S. J., and Balogh, A.: Ulysses field and plasma observations of magnetic holes in the solar wind and their relation to mirror-mode structures, J. Geophys. Res., 99, 23 371-23 381, 1994. 